\title{
Acetogenins from Annona muricata as potential inhibitors of antiapoptotic proteins: a molecular modeling study
}

This article was published in the following Dove Press journal:

Drug Design, Development and Therapy

I3 April 2016

Number of times this article has been viewed

\section{Priya Antony \\ Ranjit Vijayan}

Department of Biology, College of Science, United Arab Emirates University, Al Ain, Abu Dhabi,

United Arab Emirates
Correspondence: Ranjit Vijayan Department of Biology, College of Science, United Arab Emirates University, PO Box I555I, Al Ain, Abu Dhabi, United Arab Emirates

Tel +97। 37136302

Email ranjit.v@uaeu.ac.ae
Abstract: Apoptosis is a highly regulated process crucial for maintaining cellular homeostasis and development. The B-cell lymphoma 2 (Bcl-2) family of proteins play a crucial role in regulating apoptosis. Overexpressed Bcl-2 proteins are associated with the development and progression of several human cancers. Annona muricata is a tropical plant that belongs to the Annonaceae family and is well known for its anticancer properties. In this study, molecular docking and simulations were performed to investigate the inhibitory potential of phytochemicals present in A. muricata against antiapoptotic proteins of the Bcl-2 family including Bcl-2, B-cell lymphoma extra-large (Bcl-Xl), and Mcl-1. Docking results revealed that the acetogenins, such as annomuricin $\mathrm{A}$, annohexocin, muricatocin $\mathrm{A}$, annomuricin-D-one, and muricatetrocin $\mathrm{A} / \mathrm{B}$, exhibited strong binding interactions with $\mathrm{Bcl}-\mathrm{X} 1$ when compared to $\mathrm{Bcl}-2$ and $\mathrm{Mcl}-1$. Binding score and interactions of these acetogenins were notably better than those of currently available synthetic and natural inhibitors. Molecular dynamics simulations of the top-scoring lead molecules established that these molecules could bind strongly and consistently in the active site of $\mathrm{Bcl}-\mathrm{Xl}$. These results suggest that acetogenins could be explored as selective natural inhibitors of Bcl-Xl that could assist in promoting the intrinsic pathway of apoptosis.

Keywords: apoptosis, molecular docking, molecular dynamics, phytocompounds, drug discovery

\section{Introduction}

Ever since the discovery of apoptosis in early 1970 by Kerr et al, ${ }^{1}$ it has been one of the most explored biological processes in the area of molecular research. Apoptosis is a highly regulated and selective process crucial for maintaining cellular homeostasis and development in multicellular organisms. ${ }^{2}$ It plays an indispensable role in shaping various body parts in early embryonic development and also regulates cellular equilibrium by controlling cell number and proliferation. It also serves as a defense mechanism by discarding defective cells that have undergone major DNA damage and cannot be repaired. ${ }^{3,4}$ Apoptosis is mainly executed either by the extrinsic pathway/death receptors or by the intrinsic/mitochondrial pathway with the activation of caspases. ${ }^{5,6}$

Extrinsic pathway is evoked when cytokine ligands bind to cell-surface death receptors of the tumor necrosis factor receptor family. This binding initiates a series of events that eventually result in the catalytic activation of procaspase- 8 into caspase- 8 . Activation of caspase- 8 triggers the executional phase of apoptosis. Internal stimuli such as DNA damage, heat, virulence factors, oxidative stress, and growth factor deprivation will take the intrinsic pathway. These stimuli increase the permeability of the outer mitochondrial membrane (OMM) and release cytochrome $\mathrm{C}$ into the cytoplasm, which 
further promote caspase-9 activation and lead to apoptosis. ${ }^{2,7,8}$ Cells experiencing apoptosis will exhibit several morphological and biochemical changes such as cellular shrinkage, loss of membrane symmetry, membrane blebbing, DNA breakdown, protein cleavage, nuclear condensation, and fragmentation, and eventually these apoptotic bodies will be engulfed by phagocytes. The members of B-cell lymphoma 2 (Bcl-2) family of proteins are key regulators of the intrinsic pathway, and their dynamic binding interplay determines whether the cell survives or dies. ${ }^{9}$ Failure to maintain the delicate balance between cell survival and cell death will result in oncogenesis and chemoresistance. ${ }^{10,11}$ Due to their conflicting functions, members of the Bcl-2 family have been grouped into three major classes: prosurvival, proapoptotic, and Bcl-2 homology 3 (BH3)-only proteins. Bcl-2, B-cell lymphoma extra-large (Bcl-Xl), myeloid leukemia 1 (Mcl-1), B-cell lymphoma-w (Bcl-w), B-cell lymphoma 2-related protein (A1), and Bcl-B comprise the group of prosurvival or antiapoptotic proteins that protect the cell from various cytotoxic conditions by inhibiting proapoptotic proteins. ${ }^{12}$ Major proapoptotic proteins are the Bcl-2 antagonist/killer (Bak) and Bcl-2-associated protein X (Bax) that promote cell death via OMM permeabilization. This causes the release of apoptogenic molecules into the cytosol followed by caspase activation. ${ }^{13,14} \mathrm{BH} 3$-only proteins such as BIM, BID, BAD, HRK, BIK, PUMA, and NOXA possess conserved BH3 domains by which they interact with either proapoptotic or antiapoptotic proteins to promote apoptosis. They act as initial sensors of apoptotic signals that arise from various cellular responses such as DNA damage, endoplasmic reticulum stress, and growth factor deprivation. ${ }^{15}$ Despite these opposing functions, all Bcl-2 family members share a conserved amino acid sequence motif LXXGD (where $\mathrm{X}$ is any amino acid) known as the $\mathrm{BH} 3$ motif. Most antiapoptotic $\mathrm{Bcl}-2$ proteins contain four distinct Bcl-2 homology $(\mathrm{BH})$ domains, namely, $\mathrm{BH} 1, \mathrm{BH} 2, \mathrm{BH}$, and $\mathrm{BH} 4$, which fold into a hydrophobic groove to which $\mathrm{BH} 3$ proteins bind. The hydrophobic membrane spanning domain of these proteins helps them to get embedded in the OMM, endoplasmic reticulum membrane, and the nuclear envelope. ${ }^{16,17}$ Structural analysis reveals that the proapoptotic and $\mathrm{BH} 3$-only proteins interact with the hydrophobic binding pockets (P1-P4), which constitute a large groove on the surface of Bcl-2 family members (igure S1A) $){ }^{18}$ Binding in P2 and P4 pockets is crucial for the strong binding of polypeptides on the surface of Bcl-2 proteins.

The Bcl-2 family of proteins are important regulators of the apoptotic pathway, and overexpression of $\mathrm{Bcl}-2$ proteins is a usual scenario in various malignant tumors and chemoresistance. ${ }^{19}$ Altered Bcl-2 members are involved in the development of numerous cancers by inhibiting the apoptotic pathway. ${ }^{20,21}$ Upregulation of $\mathrm{Bcl}-2$ proteins has been reported to inhibit Bax-mediated apoptosis and induction of chemoresistance in cancer cells. ${ }^{22,23}$ This association of Bcl-2 proteins in tumor initiation, disease progression, and drug resistance makes them candidate targets in anticancer therapy. ${ }^{24}$ Finding and developing efficient inhibitor molecules for antiapoptotic proteins is a longstanding goal in tumor biology.

Since the dawn of medicine, natural products derived from medicinal plants and herbs have been used for treating various disease conditions. Phytocompounds, or compounds from plants, are once again achieving prominence in modern medicine due to their fewer side effects and cost effectiveness. A vast majority of commercially available drugs have been derived from plants. For example, vinblastine and vincristine from Catharanthus roseus (periwinkle) and taxol from the bark of Taxus brevifolia (Pacific yew) are used for treating various forms of cancers including leukemia, lymphomas, as well as breast, lung, and testicular cancers. ${ }^{25,26}$ Medicinal plants are rich sources of anticancerous compounds.

In this study, a widely studied plant, Annona muricata, was considered. ${ }^{27}$ It is commonly known as soursop, graviola, or pawpaw and belongs to the family Annonaceae. It is a tree widely found in tropical and subtropical areas of the world. In traditional medicine, these plants are extensively used for treating a wide array of diseases. ${ }^{28,29}$ It was reported that an aqueous leaf and bark extract of $A$. muricata produced hypoglycemic effect by improving the pancreatic insulin level in streptozotocin-induced diabetic rats. ${ }^{30,31}$ The ethanolic leaf extract of $A$. muricata has been shown to possess anti-inflammatory and anti-arthritic activity in animal models. ${ }^{32}$ Phytochemical analysis indicates the presence of various compounds such as acetogenins, alkaloids, flavonoids, glycosides, saponins, tannins, and carbohydrates in this plant. ${ }^{33}$ Acetogenins are white waxy derivatives of long-chain fatty acids (C32 or C34) derived through the polyketide pathway and are exclusively found in the Annonaceae family. ${ }^{34}$ They share a common structural skeleton characterized by an $\alpha, \beta$-unsaturated- $\gamma$ lactone moiety and a hydroxylated tetrahydrofuran (THF) ring joined by a long alkyl chain and a long hydrophobic tail. ${ }^{35}$ These chemicals are well known for their cytotoxic effects on various cancer cell lines and are potent inhibitors of NADH-ubiquinone oxidoreductase (mitochondrial complex I). ${ }^{36}$ It has also been shown that an ethyl acetate 
extract of $A$. muricata leaves induces apoptosis in cancer cell lines by mediating the nuclear factor $\kappa \mathrm{B}$ pathway. ${ }^{37}$ Targeting antiapoptotic proteins by small-molecule inhibitors is challenging in cancer biology research due to the difficulties in targeting many protein-protein interaction sites. ${ }^{38}$ However, inhibitors such as navitoclax and venetoclax have been developed to inhibit Bcl-2 proteins. Navitoclax is the first orally bioavailable drug currently in Phase II clinical trials. ${ }^{39,40}$ Natural polyphenols such as quercetin and apogossypol also exhibit significant inhibitory action against Bcl-2 proteins. Quercetin is a dietary polyphenol found in many plants, and apogossypol is a derivate of gossypol found in cotton plant. ${ }^{41-43}$

Computational methods such as molecular docking and molecular dynamics (MD) have been used extensively to identify new lead compounds in the field of drug discovery. ${ }^{44-46}$ The present study was designed to investigate whether the phytocompounds present in A. muricata could interact with the antiapoptotic proteins, such as Bcl-2, Bcl-X1, and Mcl-1, by analyzing their binding interactions and stability through computational approaches including molecular docking and simulation. For a comparative analysis, the potent Bcl-2 inhibitor navitoclax and the natural inhibitors quercetin and apogossypol were used as controls.

\section{Materials and methods Preparation of protein and ligand structures}

In order to ensure the validity of the results, two structures of each of the proteins - Bcl-2, Bcl-X1, and Mcl-1 - were used. Three-dimensional (3D) structures of these proteins were retrieved from the Protein Data Bank (Table 1). ${ }^{47}$ The protein structures obtained were preprocessed using the Protein Preparation Wizard of Schrödinger Maestro. This step was performed to remove unwanted water molecules, add and optimize hydrogen bonds, simplify multimeric complexes, create disulfide bonds, adjust formal charges and bond orders of atoms that are attached to metal ions and cofactors, fix the

Table I Antiapoptotic proteins from the Bcl-2 family used in this study

\begin{tabular}{llll}
\hline No & Protein target & PDB ID & Resolution (A) \\
\hline I & B-cell lymphoma & 3ZLR & 2.03 \\
& extra-large (Bcl-XI) & $4 \mathrm{QVX}$ & 2.10 \\
2 & B-cell lymphoma 2 (Bcl-2) & 2W3L & 2.10 \\
& & $4 \mathrm{AQ3}$ & 2.40 \\
3 & Myeloid leukemia I (Mcl-I) & $4 \mathrm{WMR}$ & 1.70 \\
& & $3 \mathrm{WIY}$ & 2.15 \\
\hline
\end{tabular}

Abbreviation: PDB, Protein Data Bank. orientation of misoriented groups, and, finally, optimize and refine the structure for further analysis. ${ }^{48}$

From the literature, ${ }^{28,29} 65$ natural compounds from A. muricata were identified, and structures of these compounds were retrieved from PubChem and Chemspider databases (Table S1). ${ }^{49}$ For a comparative study, Bcl-2 inhibitor navitoclax (ABT 263) and the phytochemical inhibitors quercetin and apogossypol were also retrieved and processed. ${ }^{50}$ Schrödinger's LigPrep was used to prepare the structures of these ligands, which involved the conversion of ligand two-dimensional (2D) structures to 3D, addition of hydrogen atoms, generation of various ionization states and tautomers, and, lastly, optimization of geometries. ${ }^{51}$ A total of 378 stereoisomers were generated from these ligands, which were used for the docking study.

\section{Determination of active site and grid generation}

Based on the binding of proapoptotic proteins and $\mathrm{BH} 3-$ only proteins, functional residues involved in the active site regions of antiapoptotic proteins were determined. ${ }^{52}$ A receptor grid was generated by incorporating all these functional residues. The van der Waals scaling factor for nonpolar atoms and partial charge cutoff values were set to the default values of 1.0 and 0.25 , respectively. Rotation of hydroxyl groups in the active site residues was allowed to produce the most favorable interactions. While generating the receptor grid, no constraints were used. The rest of the parameters were set to default. The OPLS 2005 force field was employed for modeling the interactions. ${ }^{53}$

\section{Molecular docking}

Molecular docking of the prepared targets and ligands was carried out using Schrödinger's Glide. It uses a series of hierarchical filters for searching favorable interactions of ligands in the active site of proteins. The extra precision (XP) dock scoring function was employed in the analysis. ${ }^{54}$ Flexible ligand sampling was used to generate conformers during docking. To soften the nonpolar parts of ligands, values for the scaling factor and partial charge cutoff were set as 0.80 and 0.15 , respectively. In the entire docking procedure, no constraints were used, and 100 docking poses were generated for each ligand. To improve the geometry of the poses, post-docking minimization was also carried out. Top compounds were ranked based on GlideScore, which is the sum of all XP terms. ${ }^{55}$ The binding pattern and interactions of these compounds were visualized using the Glide XP visualizer. ${ }^{56}$ The binding energy, based on molecular 
mechanics generalized Born surface area, was calculated using Schrödinger's Prime. ${ }^{57}$

\section{MD simulations}

Top-scoring molecules were subjected to MD simulation for a period of 100 nanoseconds using Desmond to investigate the stability and dynamic interactions of these compounds in their binding site.$^{58}$ For each docked complex, an orthorhombic box was made with a box wall distance of $10 \AA$, and the system was solvated with single point charge water molecules.${ }^{59}$ Neutralization of the entire system was achieved by adding six $\mathrm{Na}^{+}$ions and using a salt concentration of $0.15 \mathrm{M}$. The OPLS 2005 force field was used to model all atoms and their interactions. The initial step involved energy minimization of the prepared system for 2,000 steps using the steepest descent method. For equilibrating the entire system, Desmond's default protocol was used. After this, MD simulations were performed for a period of 100 nanoseconds at $300 \mathrm{~K}$ temperature and 1.01325 bar pressure.$^{60}$ For this, the Nosé-Hoover chain thermostat was used with a relaxation time of $1 \mathrm{ps}$ and the isotropic Martyna-Tobias-Klein barostat was used with a relaxation time of 2 ps. ${ }^{61,62}$ For short-range Coulombic interactions, a $9 \AA$ cutoff was set, while longrange interactions were evaluated using the smooth particle mesh Ewald method.$^{63}$ At every 5 ps, frames were captured to form the simulation trajectory. After the entire simulation, root-mean-square deviation and root-mean-square fluctuation of protein-ligand complexes were calculated with reference to the first frame of the simulation trajectory. Hydrogen bonds and other nonbonded interactions formed between the protein and the ligand throughout the simulation were also analyzed. Rescoring of the simulated docked complexes was performed using Glide by extracting a frame every 10 nanoseconds from the 100 nanoseconds simulation trajectory.

\section{Results and discussion}

To explore the possible binding patterns and interactions of phytocompounds to Bcl-2, Bcl-Xl, and Mcl-1, molecular docking and simulation approaches were employed. The 65 ligands used for XP docking were evaluated using GlideScore and binding energies. The results were analyzed based on their interactions in the active site of these antiapoptotic proteins. Table 2 lists the top-ranked molecules in Bcl-Xl and Bcl-2 along with the interacting residues. In Bcl-Xl, five acetogenins, namely, annomuricin A, annohexocin, muricatocin $\mathrm{A}$, muricatetrocin $\mathrm{A} / \mathrm{B}$, annomuricinD-one (Figure 1A), exhibited high GlideScore ranging from $-13.00 \mathrm{kcal} / \mathrm{mol}$ to $-11.21 \mathrm{kcal} / \mathrm{mol}$ in one structure (3ZLR) and from $-12.59 \mathrm{kcal} / \mathrm{mol}$ to $-10.60 \mathrm{kcal} / \mathrm{mol}$ in the other (4QVX). In Bcl-2 proteins, the acetogenins muricin E, annomuricin A, and annohexocin came on top with GlideScores ranging from $-9.32 \mathrm{kcal} / \mathrm{mol}$ to $-8.80 \mathrm{kcal} / \mathrm{mol}$ in $2 \mathrm{~W} 3 \mathrm{~L}$ and from $-9.89 \mathrm{kcal} / \mathrm{mol}$ to $-9.58 \mathrm{kcal} / \mathrm{mol}$ in $4 \mathrm{AQ} 3$.

In Mcl-1, the phytochemicals failed to generate consistent interactions in the two structures, indicating low specificity (Table S2). Even though Bcl-2 exhibited consistent interactions in both structures, from the GlideScores it is evident that acetogenins interacted much better with $\mathrm{Bcl}-\mathrm{Xl}$ than Bcl-2. These molecules interacted with key residues in the active site by establishing hydrogen bonding and

Table 2 GlideScores and residues in $\mathrm{Bcl}-\mathrm{XI}$ and $\mathrm{Bcl}-2$ that formed hydrogen bonds with the shortlisted acetogenins

\begin{tabular}{|c|c|c|c|c|}
\hline Protein & PDB ID & Ligands & GlideScore (kcal/mol) & Interacting residues \\
\hline \multirow[t]{10}{*}{$\mathrm{Bcl}-\mathrm{XI}$} & $3 Z L R$ & Annomuricin A & -13.00 & Ser 106, Arg132, Asp 133, Asn 136, Argl39 \\
\hline & & Annohexocin & -12.90 & Ser 106, Asp 107, Leul08, Leul30, Argl 39 \\
\hline & & Muricatocin A & -12.54 & Arg102, Ser 106, Leu 108, Leul30 \\
\hline & & Annomuricin-D-one & -11.37 & Ser 106, Arg132, Asp 133, Asn 136, Arg139 \\
\hline & & Muricatetrocin A/B & $-\mid 1.21$ & Argl02, Ala |42, Asn I36, Argl 39 \\
\hline & $4 Q V X$ & Annomuricin $\mathrm{A}$ & -12.59 & Ser 106, Leul 08, Leul30 \\
\hline & & Annohexocin & -12.54 & Arg102, Ser 106, Leu 108, Leul30, Serl45 \\
\hline & & Muricatocin A & -12.58 & Ser106, Aspl07, Asn 136, Argl39 \\
\hline & & Annomuricin-D-one & -11.12 & $\operatorname{Arg} 102$, Leu 108, GluI29, Arg132, Argl39 \\
\hline & & Muricatetrocin A/B & -10.60 & Arg102, Ser106, Arg139 \\
\hline \multirow[t]{6}{*}{$\mathrm{Bcl}-2$} & $2 W 3 L$ & Muricin E & -9.32 & Asp99, Leu96, Arg105 \\
\hline & & Annohexocin & -9.17 & Gly77, Arg88, His79, Glu95 \\
\hline & & Annomuricin $\mathrm{A}$ & -8.80 & Glu95, Leu96, Arg105 \\
\hline & 4AQ3 & Annomuricin $\mathrm{A}$ & -9.89 & Tyr67, Asn 102, Arg105, Tyrl6I \\
\hline & & Annohexocin & -9.73 & Tyr67, Arg105 \\
\hline & & Muricin E & -9.58 & Asp62, Arg66, Asn 102, Ala 108 \\
\hline
\end{tabular}

Abbreviations: Bcl-XI, B-cell lymphoma extra-large; Bcl-2, B-cell lymphoma 2; PDB, Protein Data Bank. 


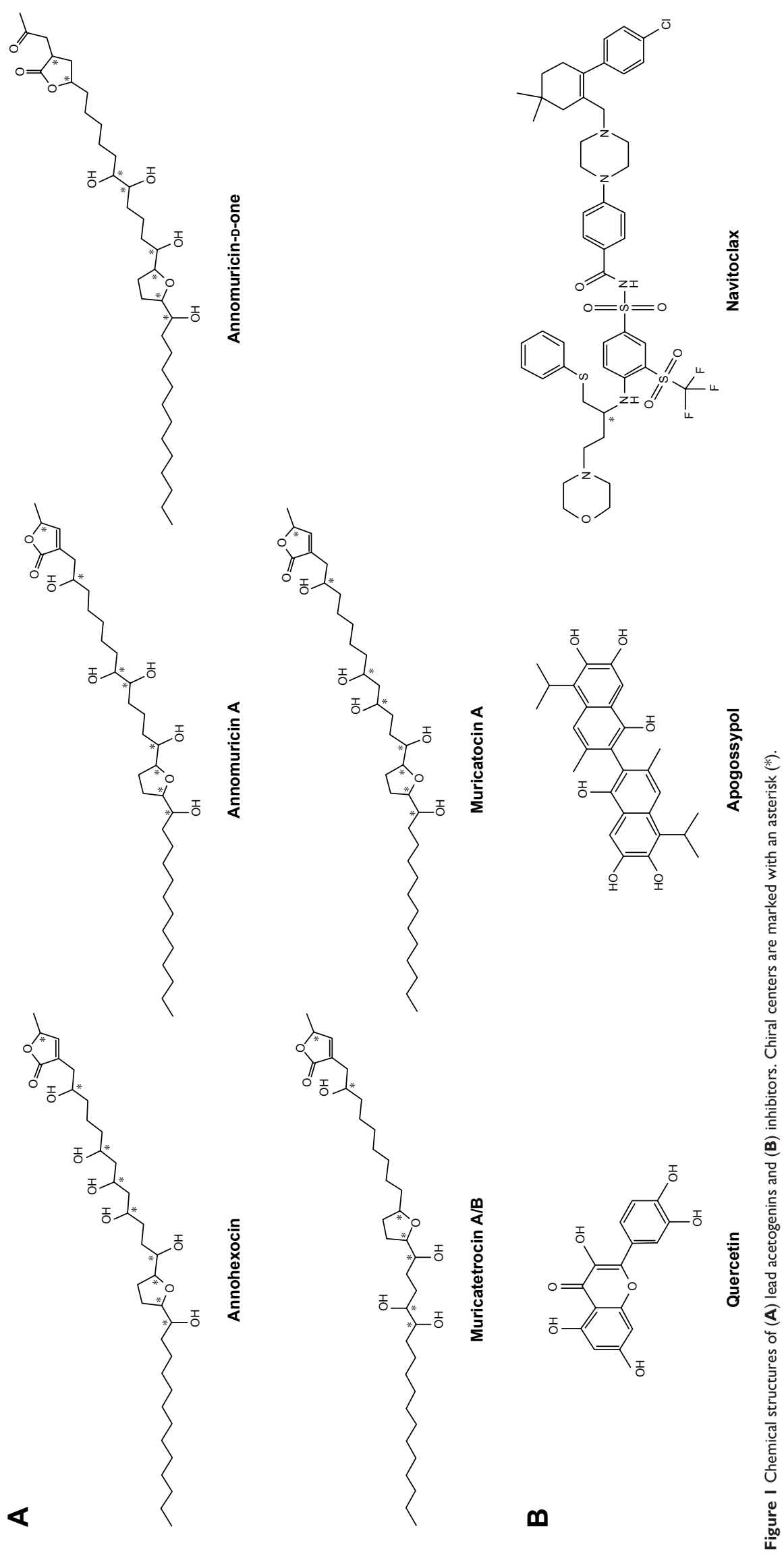


hydrophobic interactions. Thus, from the GlideScores and binding energies, acetogenins appear to have a higher specificity for Bcl-X1 than Bcl-2 or Mcl-1 (Tables 2 and $\underline{\mathrm{S} 2}$ ).

\section{Binding interactions of acetogenins with $\mathrm{Bcl}-\mathrm{XI}$}

The 3D structure of antiapoptotic/prosurvival protein $\mathrm{Bcl}-\mathrm{Xl}$ (4QVX and 3ZLR) consists of a common domain formed by assembling two hydrophobic ( $\alpha 5$ and $\alpha 6$; Figure S2) and six amphipathic $\alpha$ helices $(\alpha 1-\alpha 4, \alpha 7$, and $\alpha 8) .{ }^{64}$ Four amphipathic helices fold to form a long hydrophobic groove of nearly $20 \AA$ in length (Figure S1A). Structural insights reveal that high-affinity binding of proapoptotic protein, and $\mathrm{BH} 3$-only proteins is mediated by interactions in the P2 and P4 pockets of the hydrophobic groove and also by electrostatic interactions between conserved arginine and aspartic acid residues of proapoptotic and prosurvival proteins, respectively. ${ }^{24,52}$ The $\mathrm{P} 2$ and $\mathrm{P} 4$ pockets are lined by residues Glu96, Tyr101, Ser106, Asp107, Leu108, Arg139, and Tyr195. ${ }^{24}$ The P2 hydrophobic pocket is crucial for tight binding of ligand molecules due to its deep and plastic cavity. ${ }^{65}$ Analysis of the binding pose of annomuricin A in 3ZLR showed that it interacted with the protein by forming six hydrogen bonds and numerous hydrophobic interactions with the critical residues seen in the active site. Annomuricin A is present in the leaves and pericarp of A. muricata, and it has an $\alpha, \beta$-unsaturated- $\gamma$-lactone moiety and a monoTHF ring with five hydroxyl groups (Figure 1A). ${ }^{28,66}$ The $\alpha, \beta$-unsaturated- $\gamma$-lactone moiety of annomuricin A was buried in the P2 pocket, and the hydroxyl group adjacent to this moiety formed a hydrogen bond with the important Ser106 in the P2 pocket that is required for tight binding (Figure S1B) ${ }^{65}$ The remaining four hydroxyl groups in this molecule interacted with Arg132, Asp133, Asn136, and Arg139 by forming hydrogen bonds. The long hydrophobic tail of the molecule extended into the P4 pocket and formed interactions with several hydrophobic residues, including Tyr101 and Tyr195 (Figures 2B and S3A). ${ }^{24}$ Entropic penalty imposed by restraining the ligand in the binding site was overcome by the highly favorable lipophilic and hydrogen-bond interactions in the binding site. A similar binding pattern was also observed in the 4QVX-annomuricin A complex. The hydroxyl groups adjacent to the THF ring region and the alkyl chain formed hydrogen bonds with the key residues such as Ser106, Leu108, and Leu130 in the pockets (Figures $3 \mathrm{~B}$ and $\underline{\mathrm{S} 4 \mathrm{~A}}$ ). In the two Bcl-Xl structures considered, annomuricin A exhibited GlideScores of $-13.00 \mathrm{kcal} / \mathrm{mol}$ and $-12.59 \mathrm{kcal} / \mathrm{mol}$, respectively.
Numerous hydrophobic contacts contributed to its binding to Bcl-X1 (Figures S3A and S4A).

Annohexocin, present in the leaves of $A$. muricata, is a C-35 mono-THF acetogenin with six hydroxyl groups. ${ }^{28,67}$ In the 3ZLR-annohexocin complex, the hydroxyl groups in the compound formed six hydrogen bonds with Ser106, Asp107, Leu108, Leu130, and Arg139. The $\alpha, \beta$-unsaturated$\gamma$-lactone moiety of annohexocin occupies the $\mathrm{P} 2$ pocket, and the hydrophobic tail region of the molecule extends into the $\mathrm{P} 4$ pocket by forming hydrophobic contacts. The interactions with these important residues in the active site contribute to their high binding score (Figures $2 \mathrm{C}$ and $\underline{\mathrm{S} 3 \mathrm{~B}}$ ). Similar interactions were also observed in the 4QVX-annohexocin complex. The hydroxyl groups in the alkyl chain formed strong hydrogen bonds with Arg102, Ser106, Leu108, and Ser145, while the hydroxyl group adjacent to THF ring interacted with Leu130. The hydrophobic tail extended into the $\mathrm{P} 4$ region (Figures $3 \mathrm{C}$ and $\underline{\mathrm{S}} 4 \mathrm{~B}$ ).

Muricatocin A, found in the leaves of A. muricata, is a mono-THF acetogenin with five hydroxyl groups. ${ }^{68}$ It interacted with 3ZLR by forming five hydrogen bonds with Arg102, Ser106, Leu108, and Leu130 (Figures 2D and $\underline{\mathrm{S} 3 \mathrm{C}}$ ). In the 4QVX-muricatocin A complex, it formed five hydrogen bonds with Ser106, Asp107, Asn136, and Arg139 (Figures 3D and S4C).

In both structures, the three molecules, namely, annomuricin A, annohexocin, and muricatocin A, displayed consistent interactions in the active site, with GlideScores ranging from $-13.00 \mathrm{kcal} / \mathrm{mol}$ to $-12.58 \mathrm{kcal} / \mathrm{mol}$. All three structures are $\mathrm{C} 35$ mono-THF ring acetogenins with identical spacing between the $\alpha, \beta$-unsaturated- $\gamma$-lactone moiety and the THF ring. Even though there is a minor variation in the number and position of the hydroxyl groups, all three bound strongly in the P2 pocket by forming hydrogen bonds with critical residues such as Ser106, Asp107, and Leu108. This suggests that the number and position of hydroxyl groups do not have a major impact on the binding efficiency of these compounds (Figures S3 and S4). Furthermore, this is supported by molecular mechanics generalized Born surface area calculations, which show that lipophilic interactions contribute the most to the total binding energy (Table S3).

Compared to the aforementioned three compounds, annomuricin-D-one and muricatetrocin A/B displayed lower GlideScore in both Bcl-X1 structures. These two compounds consist of a mono-THF ring with four hydroxyl groups (Figure 1A). ${ }^{69,70}$ The interactions between Bcl-Xl and these compounds are shown in Figures $2 \mathrm{E}$ and F, 3E and F, S3D and $\underline{E}$, and $\underline{S 4 D}$ and $\underline{E}$. The length of the alkyl chain that connects 
A

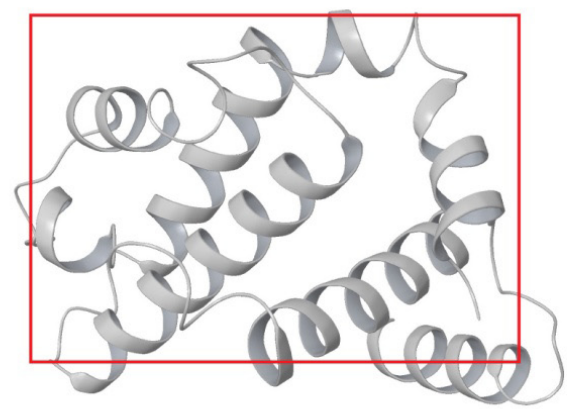

C
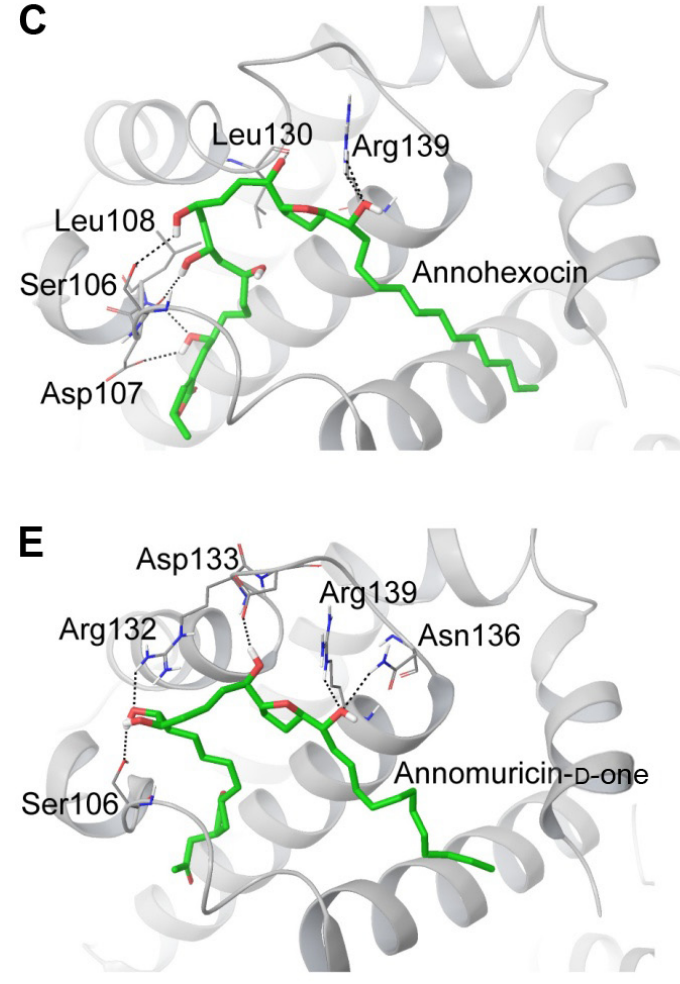

B
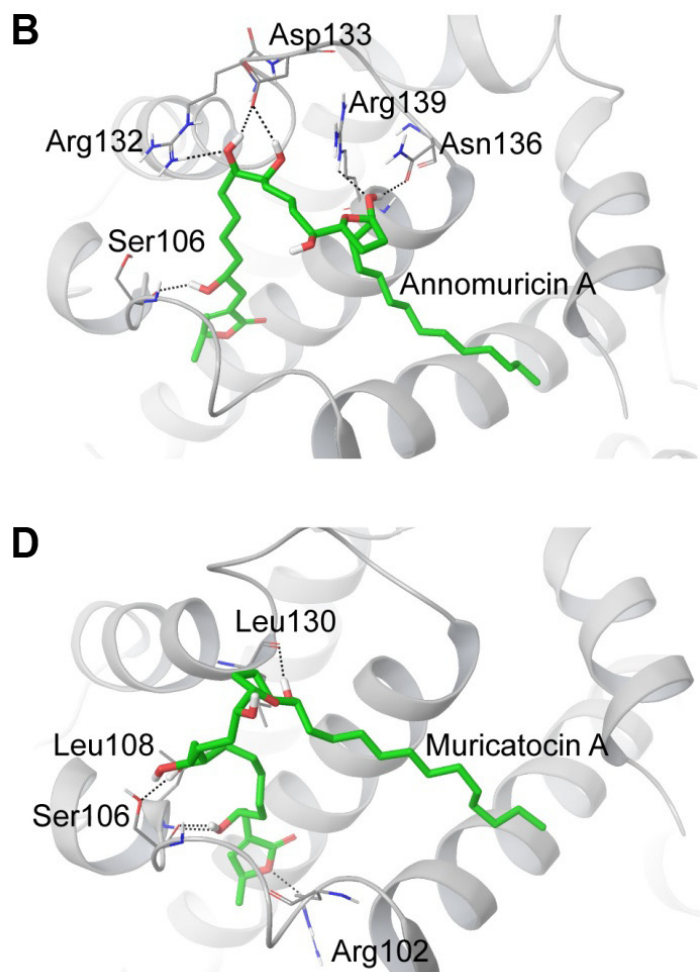

$\mathbf{F}$

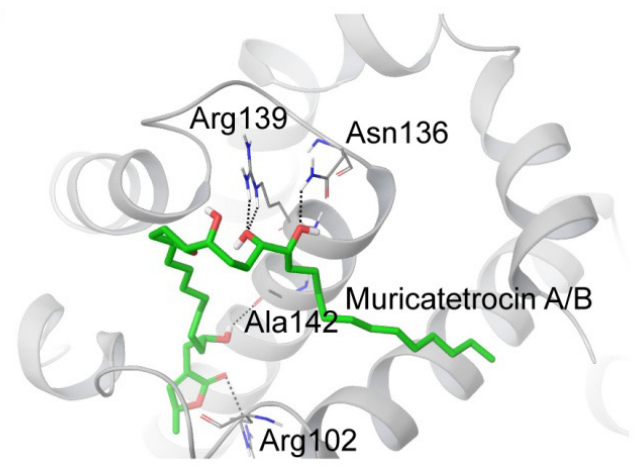

Figure 2 Binding interactions of acetogenins in the active site of $\mathrm{Bcl}-\mathrm{XI}$ (3ZLR).

Notes: (A) Bcl-XI protein structure with the region shown in (B-F) boxed in red. Interaction of Bcl-XI with (B) annomuricin A, (C) annohexocin, (D) muricatocin A, (E) annomuricn-D-one, and (F) muricatetrocin A/B.

Abbreviation: Bcl-XI, B-cell lymphoma extra-large.

the $\alpha, \beta$-unsaturated- $\gamma$-lactone moiety and THF ring is shorter when compared to those in annomuricin A, annohexocin, and muricatocin A. This suggests that the length of alkyl chain plays a role in the binding efficiency of these compounds; longer the alkyl chain, the stronger the interactions.

Since deregulation of apoptosis is a common scenario in many forms of cancers, several drugs have been developed to inhibit the Bcl-2 family proteins. It was reported that the natural molecules quercetin and apogossypol also interact directly with $\mathrm{Bcl}-\mathrm{Xl}$ and trigger apoptotic activity. For a comparative analysis, the Bcl-Xl inhibitor navitoclax and these natural molecules were used as controls (Figure 1B). ${ }^{39,40}$ Docking results (Figure S5) showed that acetogenins exhibited better
GlideScore than these inhibitors (Table 3). Pharmacokinetic properties computed for the acetogenins using Schrödinger QikProp were also notably better than those of navitoclax (Table S4). These acetogenins have also been shown to exhibit significant cytotoxicity against several cancer cell lines including MCF-7 (breast), A549 (lung), HT-29 (colon), and MIA PaCa-2 (pancreatic). ${ }^{28,66-70}$

\section{MD simulations}

In order to analyze structural dynamics and stability of annomuricin $\mathrm{A}$, annohexocin and muricatocin $\mathrm{A}$ bound to $\mathrm{Bcl}-\mathrm{Xl}, \mathrm{MD}$ simulations were performed for a period of 100 nanoseconds each. During the initial period of the 
A

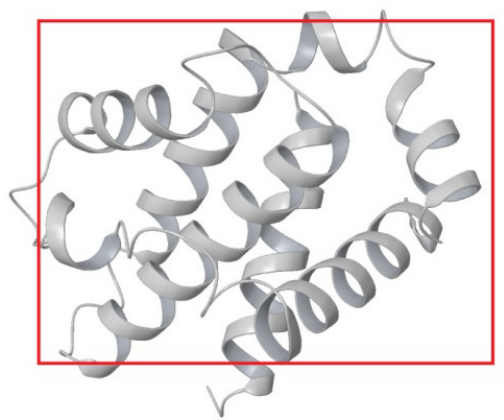

C
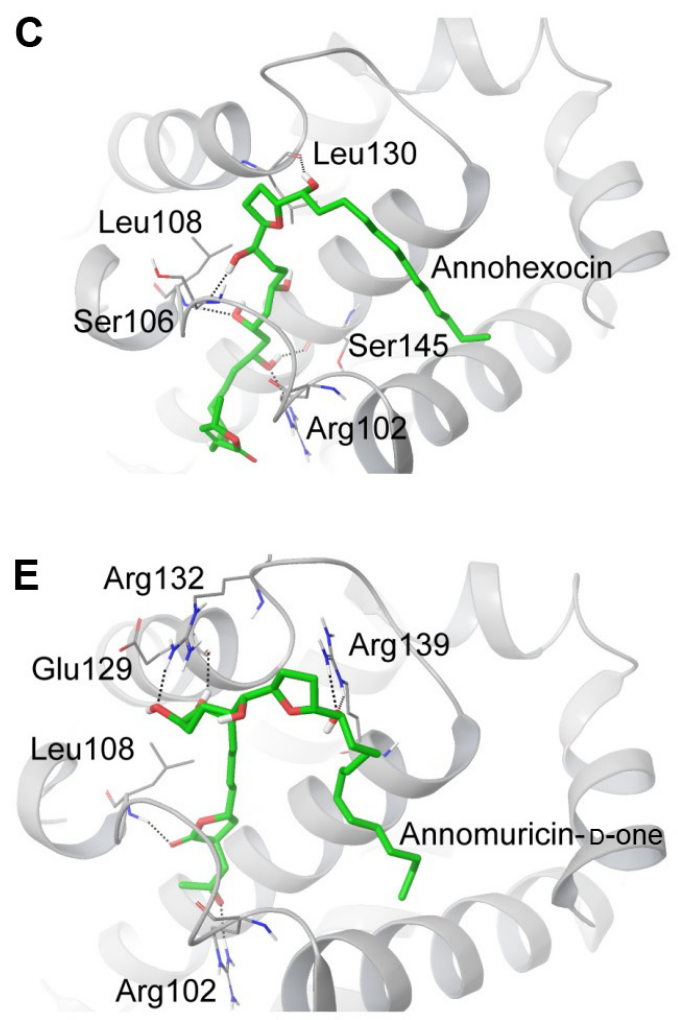

B

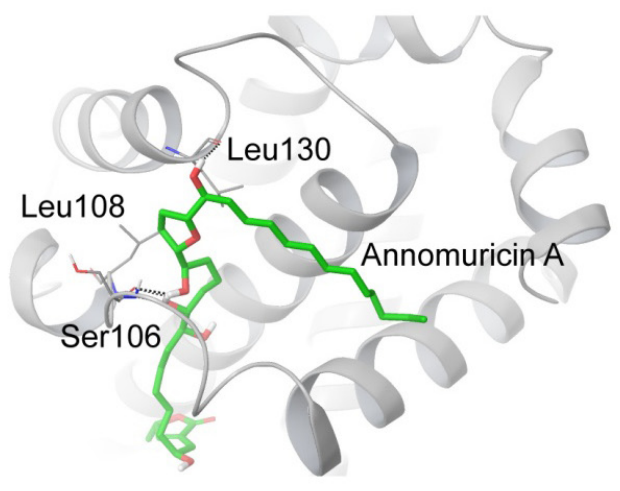

D

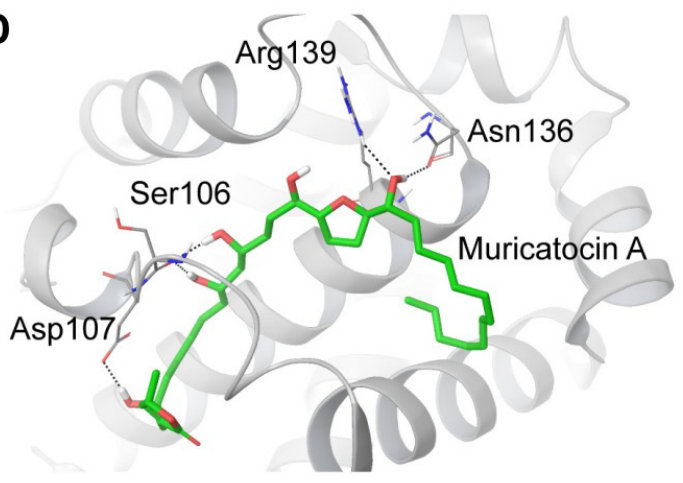

$\mathbf{F}$

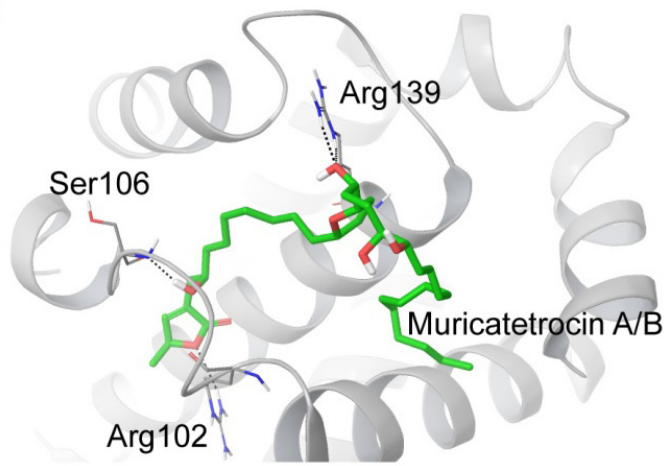

Figure 3 Binding interactions of acetogenins in the active site of $\mathrm{Bcl}-\mathrm{XI}(4 \mathrm{QVX})$.

Notes: $(\mathbf{A}) \mathrm{Bcl}-\mathrm{XI}$ protein structure with the region shown in $(\mathbf{B}-\mathbf{F})$ boxed in red. Interaction of $\mathrm{Bcl}-\mathrm{XI}$ with $(\mathbf{B})$ annomuricin $\mathrm{A}$, (C) annohexocin, (D) muricatocin $\mathrm{A}$, (E) annomuricn-D-one, and (F) muricatetrocin A/B.

Abbreviation: $\mathrm{Bcl}-\mathrm{Xl}, \mathrm{B}$-cell lymphoma extra-large.

simulations, Bcl-Xl exhibited some degree of fluctuation, indicating that the protein was undergoing structural adaptations. After this initial period, the protein structure stabilized, providing an equilibrated system. Most of the fluctuations were observed in the loops and turns, whereas the other secondary structures remained intact throughout the simulations. During the course of the simulation, annomuricin A stayed firmly in the binding pocket of Bcl-Xl by forming hydrogen bonds, hydrophobic interactions, and water bridges with the critical residues Tyr101, Ser106, Leu108, and Arg 139. Besides maintaining these crucial interactions, it also formed new interactions in the active site (Figure 4A).
Annohexocin also adapted well to the binding pocket of Bcl-Xl by maintaining the important interactions with the residues in the $\mathrm{P} 2$ pocket. Throughout the simulation, annohexocin retained the hydrogen-bond interactions with Ser106, Asp107, and Leu108 residues (Figure 4B). These sustained interactions confirmed the strong binding of the molecule in the active site of $\mathrm{Bcl}-\mathrm{Xl}$. Muricatocin A also remained in the P2 pocket by intermittently forming and breaking hydrogen bonds with Phe105, Ser106, Asp107, and Leu108 residues (Figure 4C). In all three docked complexes, water molecules assisted in forming numerous water bridges, thereby making the interactions stronger. To study how the water molecules 
Table 3 GlideScores and $\mathrm{Bcl}-\mathrm{XI}$ residues that formed hydrogen bonds with the known inhibitors

\begin{tabular}{|c|c|c|c|}
\hline PDB ID & Ligands & GlideScore (kcal/mol) & Interacting residues \\
\hline \multirow[t]{3}{*}{ 3ZLR } & Navitoclax & -8.04 & Phel05, Ser 106, Arg132, Tyr I95 \\
\hline & Quercetin & -9.46 & Phel05, Ser I06, Ala I42 \\
\hline & Apogossypol & -7.66 & Leul94 \\
\hline \multirow[t]{3}{*}{$4 Q V X$} & Navitoclax & -8.00 & Phel05, Ser 106, Glu 129, ArgI39 \\
\hline & Quercetin & -9.87 & Argl 02, Phel05, Serl06 \\
\hline & Apogossypol & -7.63 & Leul94 \\
\hline
\end{tabular}

Abbreviations: Bcl-XI, B-cell lymphoma extra-large; PDB, Protein Data Bank.

could contribute to the binding energy, frames were extracted every 10 nanoseconds from the MD simulations. These were then rescored using the same protocol as used for molecular docking. In the case of annomuricin A, average rescored
GlideScore of all frames analyzed was $-18.10 \pm 1.57 \mathrm{kcal} / \mathrm{mol}$ and the final frame produced a GlideScore of $-19.15 \mathrm{kcal} /$ mol. The average GlideScores of annohexocin and muricatocin A were $-19.67 \pm 1.50 \mathrm{kcal} / \mathrm{mol}$ and $-18.98 \pm 2.07 \mathrm{kcal} / \mathrm{mol}$,
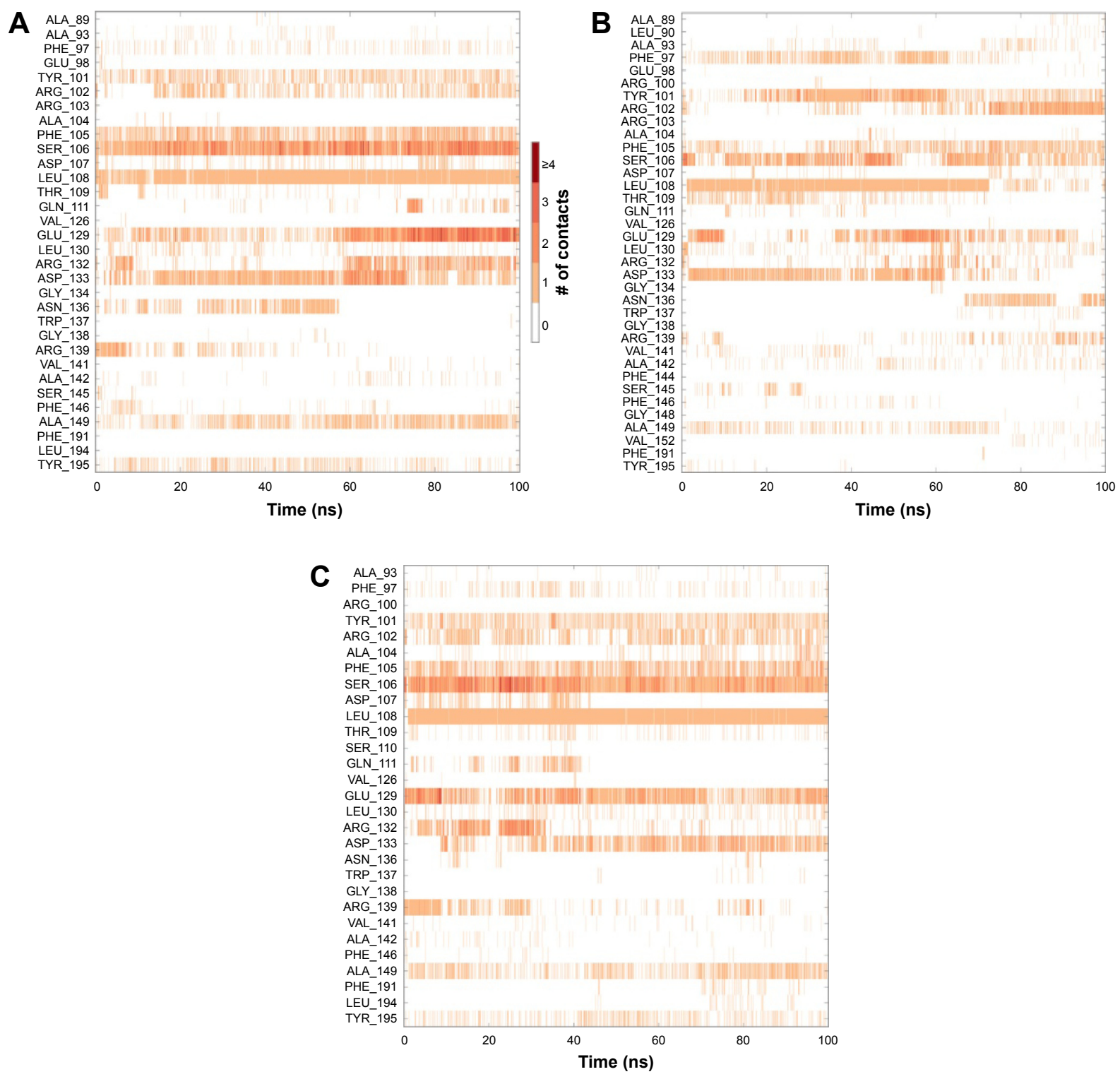

Figure 4 Residues of $\mathrm{Bcl}-\mathrm{XI}$ that interacted with the bound ligand during the course of the 100 nanoseconds simulation. Notes: (A) Annohexocin, (B) annomuricin A, and (C) muricatocin A.

Abbreviation: $\mathrm{Bcl}-\mathrm{Xl}$, B-cell lymphoma extra-large. 
respectively. All three lead molecules exhibited significant improvement from the initially docked GlideScore (Table 2). During the course of the simulation, water molecules played a crucial role by forming a number of direct interactions and bridges between the ligand and amino acids in the binding site of the protein (Figure S6). This contributed to the significant improvement in the binding scores and assisted the acetogenins to bind more favorably in the active site of Bcl-Xl. This finding highlights the significance of considering water molecules while evaluating protein-ligand interactions, especially in drug discovery.

\section{Conclusion}

Overexpression of antiapoptotic proteins is typical in many forms of cancer. In this study, molecular docking and simulations were employed to identify potential lead compounds from $A$. muricata that could be inhibitors of these antiapoptotic proteins. The results revealed that the acetogenins such as annomuricin A, annohexocin, muricatocin A, annomuricin-D-one, and muricatetrocin A/B exhibited high docking score in Bcl-Xl but not in Bcl-2 and Mcl-1. Structural and interaction analysis showed that these molecules bound stably in the active site of Bcl-X1 largely through lipohillic interactions. In comparative docking studies using natural and synthetic inhibitors of $\mathrm{Bcl}-\mathrm{Xl}$, acetogenins exhibited better binding scores and intermolecular interactions. Docking scores were also significantly improved when the protein-ligand complexes were rescored using frames from MD simulations, suggesting that water molecules play an important role in the binding site interaction. Thus, this study provides insights into how acetogenins from $A$. muricata could be the base for designing potential Bcl-Xl-selective inhibitors for the treatment of cancer.

\section{Acknowledgment}

This work was supported by an interdisciplinary research grant from the Zayed Bin Sultan Center for Health Sciences, United Arab Emirates University.

\section{Disclosure}

The authors report no conflicts of interest in this work.

\section{References}

1. Kerr JFR, Wyllie AH, Currie AR. Apoptosis: a basic biological phenomenon with wide-ranging implications in tissue kinetics. Br J Cancer. 1972;26(4):239-257.

2. Wong RS. Apoptosis in cancer: from pathogenesis to treatment. $J$ Exp Clin Cancer Res. 2011;30(1):87.

3. Saikumar P, Venkatachalam MA. Apoptosis and cell death. In: Cagle PT, Allen TC, editors. Basic Concepts of Molecular Pathology. Berlin: Springer; 2009:147-151.
4. Taylor RC, Cullen SP, Martin SJ. Apoptosis: controlled demolition at the cellular level. Nat Rev Mol Cell Biol. 2008;9(3):231-241.

5. Elmore S. Apoptosis: a review of programmed cell death. Toxicol Pathol. 2007;35(4):495-516.

6. Su Z, Yang Z, Xu Y, Chen Y, Yu Q. Apoptosis, autophagy, necroptosis, and cancer metastasis. Mol Cancer. 2015;14(1):48.

7. Ghobrial IM, Witzig ET, Adjei AA. Targeting apoptosis pathways in cancer therapy. CA Cancer J Clin. 2005;55(3):178-194.

8. Nagasaka A, Kawane K, Yoshida H, Nagata S. Apaf-1-independent programmed cell death in mouse development. Cell Death Differ. 2010; 17(6):931-941.

9. Frenzel A, Grespi F, Chmelewskij W, Villunger A. Bcl2 family proteins in carcinogenesis and the treatment of cancer. Apoptosis. 2009; 14(4):584-596.

10. Plati J, Bucur O, Khosravi-Far R. Dysregulation of apoptotic signalling in cancer: molecular mechanisms and therapeutic opportunities. $J$ Cell Biochem. 2008;104(4):1124-1149.

11. Lowe SW, Lin AW. Apoptosis in cancer. Carcinogenesis. 2000;21(3): 485-495.

12. Adams JM, Cory S. The Bcl-2 apoptotic switch in cancer development and therapy. Oncogene. 2007;26(9):1324-1337.

13. Tzifi F, Economopoulou C, Gourgiotis D, Ardavanis A, Papageorgiou S, Scorilas A. The role of BCL2 family of apoptosis regulator proteins in acute and chronic leukemias. Adv Hematol. 2012;2012:1-15.

14. Korsmeyer SJ. BCL-2 gene family and the regulation of programmed cell death. Cancer Res. 1999;59(7 Suppl):1693s-1701s.

15. Youle RJ, Strasser A. The BCL-2 protein family: opposing activities that mediate cell death. Nat Rev Mol Cell Biol. 2008;9(1): $47-59$.

16. Michels J, Kepp O, Senovilla L, et al. Functions of BCL-X L at the interface between cell death and metabolism. Int J Cell Biol. 2013;2013: 705294.

17. Shamas-Din A, Kale J, Leber B, Andrews DW. Mechanisms of action of Bcl-2 family proteins. Cold Spring Harb Perspect Biol. 2013;5(4): a008714.

18. Zhou H, Chen J, Meagher JL, et al. Design of Bcl-2 and Bcl-xL inhibitors with subnanomolar binding affinities based upon a new scaffold. J Med Chem. 2012;55:4664-4682.

19. Del Bufalo D, Biroccio A, Leonetti C, Zupi G. Bcl-2 overexpression enhances the metastatic potential of a human breast cancer line. FASEB J. 1997;11(12):947-953.

20. Yip KW, Reed JC. Bcl-2 family proteins and cancer. Oncogene. 2008; 27(50):6398-6406.

21. Akl H, Vervloessem T, Kiviluoto S, et al. A dual role for the antiapoptotic Bcl-2 protein in cancer: mitochondria versus endoplasmic reticulum. Biochim Biophys Acta. 2014;1843(10):2240-2252.

22. Teijido O, Dejean L. Upregulation of Bcl2 inhibits apoptosis-driven BAX insertion but favors BAX relocalization in mitochondria. FEBS Lett. 2010;584(15):3305-3310.

23. Dong J, Zhao YP, Zhou L, Zhang TP, Chen G. Bcl-2 upregulation induced by miR-21 via a direct interaction is associated with apoptosis and chemoresistance in MIA PaCa-2 pancreatic cancer cells. Arch Med Res. 2011;42(1):8-14.

24. Tao Z, Hasvold L, Wang L, et al. Discovery of a potent and selective BCL XL inhibitor with in vivo activity. ACS Med Chem Lett. 2014;5: 1088-1093.

25. Ahmed M, Khan RA, Khan I, Khan MR. Role of medicinal plants in oxidative stress and cancer. Sci Rep. 2013;2:64.

26. Rashed KN. Medicinal plants as a safe target for treatment of cancer. Nat Prod Chem Res. 2014;2(2):6836.

27. Yang C, Gundala SR, Mukkavilli R, Vangala S, Reid MD, Aneja R. Synergistic interactions among flavonoids and acetogenins in Graviola (Annona muricata) leaves confer protection against prostate cancer. Carcinogenesis. 2015;36(6):656-665.

28. Moghadamtousi S, Fadaeinasab M, Nikzad S, Mohan G, Ali H, Kadir H. Annona muricata (Annonaceae): a review of its traditional uses, isolated acetogenins and biological activities. Int J Mol Sci. 2015; 16(7):15625-15658. 
29. Gajalakshmi S, Vijayalakshmi S, Devi Rajeswari V. Phytochemical adn pharmacological properties of Annona muricata: a review. Int J Pharm Pharm Sci. 2012;4(2):13-16.

30. Adewole S, Caxton-Martins E. Morphological changes and hypoglycemic effects of Annona muricata Linn. (Annonaceae) leaf aqueous extract on pancreatic $\beta$-cells of streptozotocin-treated diabetic rats. Afr J Biomed Res. 2009;9(3):173-187.

31. Ahalya B, Shankar KR, Kiranmayi GVN. Exploration of antihyperglycemic and hypolipidemic activities of ethanolic extract of Annona muricata bark in alloxan induced diabetic rats. Int J Pharm Sci Rev Res. 2014;25(2):21-27.

32. Foong CP, Hamid RA. Evaluation of anti-inflammatory activities of ethanolic extract of Annona muricata leaves. Rev Bras Farmacogn. 2012; 22(6):1301-1307.

33. Vijayameena C, Subhashini G, Loganayagi M, Ramesh B. Phytochemical screening and assessment of antibacterial activity for the bioactive compounds in Annona muricata. Int J Curr Microbiol Appl Sci. 2013; 2(1):1-8.

34. Kojima N, Tanaka T. Medicinal chemistry of annonaceous acetogenins: design, synthesis, and biological evaluation of novel analogues Molecules. 2009;14(9):3621-3661.

35. Miyoshi H, Ohshima M, Shimada H, Akagi T, Iwamura H, McLaughlin JL. Essential structural factors of annonaceous acetogenins as poten inhibitors of mitochondrial complex I. Biochim Biophys Acta. 1998 1365(3):443-452.

36. Zafra-Polo MC, González MC, Estornell E, Sahpaz S, Cortes D. Acetogenins from Annonaceae, inhibitors of mitochondrial complex I. Phytochemistry. 1996;42(2):253-271.

37. Moghadamtousi SZ, Kadir HA, Paydar M, Rouhollahi E, Karimian H. Annona muricata leaves induced apoptosis in A549 cells through

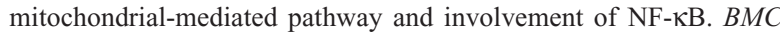
Complement Altern Med. 2014;14(1):299.

38. Mullard A. Protein-protein interaction inhibitors get into the groove. Nat Rev Drug Discov. 2012;11(3):173-176.

39. Went DM. The discovery of navitoclax, a Bcl-2 family inhibitor. In: Wendt MD, editor. Topics in Medicinal Chemistry. Protein-Protein Interactions. Vol. 8. Berlin: Springer; 2012:231-258.

40. Wong M, Tan N, Zha J, et al. Navitoclax (ABT-263) reduces Bcl-xLmediated chemoresistance in ovarian cancer models. Mol Cancer Ther. 2012;11(4):1026-1035.

41. Mantzaris MD, Hatzimichael E, Shin J, Chi S. Direct binding of Bcl 2 family proteins by quercetin triggers its pro-apoptotic activity. ACS Chem Biol. 2014;9(12):11-15.

42. Oliver CL, Miranda MB, Shangary S, Land S, Wang S, Johnson DE. (-)-Gossypol acts directly on the mitochondria to overcome Bcl-2- and Bcl-X(L)-mediated apoptosis resistance. Mol Cancer Ther. 2005;4(1): 23-31.

43. Wei J, Kitada S, Rega MF, et al. Apogossypol derivatives as antagonists of anti-apoptotic Bcl-2 family proteins. Mol Cancer Ther. 2009;8(4): 904-913.

44. Antony P, Vijayan R. Identification of novel aldose reductase inhibitors from spices: a molecular docking and simulation study. PLoS One. 2015;10(9):e0138186.

45. Singh SP, Konwar BK. Molecular docking studies of quercetin and its analogues against human inducible nitric oxide synthase. Springerplus. 2012;1(1):69.

46. Azam F, Amer AM, Abulifa AR, Elzwawi MM. Ginger components as new leads for the design and development of novel multi-targeted anti-Alzheimer's drug: a computational investigation. Drug Des Devel Ther. 2014;8:2045-2059.

47. Sussman JL, Lin D, Jiang J, et al. Protein Data Bank (PDB): database of three-dimensional structural information of biological macromolecules. Acta Crystallogr D Biol Crystallogr. 1998;54(Pt 6 Pt 1): 1078-1084.

48. Schrödinger. Schrödinger Suite 2014-1 Protein Preparation Wizard; Epik version 3.0, Schrödinger, LLC, New York, NY, 2014; Impact version 6.5, Schrödinger, LLC, New York, NY, 2014; Prime version 3.8, Schrödinger, LLC, New York, NY, 2014.
49. Wang Y, Xiao J, Suzek TO, Zhang J, Wang J, Bryant SH. PubChem: a public information system for analyzing bioactivities of small molecules. Nucleic Acids Res. 2009;37:623-633.

50. Wilson WH, Connor OAO, Czuczman MS, et al. Safety, pharmacokinetics, pharcodynamics, and activity of navitoclax, a targeted high affinity inhibitor of BCL-2, in lymphoid malignancies. Lancet Oncol. 2011;11(12):1149-1159.

51. Schrödinger. LigPrep, version 3.2. Schrödinger, LLC, New York, NY, 2014.

52. Lessene G, Czabotar PE, Sleebs BE, et al. Structure-guided design of a selective BCL-XL inhibitor. Nat Chem Biol. 2013;9(6):390-397.

53. Banks JL, Beard HS, Cao Y, et al. Integrated modeling program, applied chemical theory (impact). J Comput Chem. 2005;26(16): 1752-1780.

54. Friesner RA, Murphy RB, Repasky MP, et al. Extra precision glide: docking and scoring incorporating a model of hydrophobic enclosure for protein-ligand complexes. J Med Chem. 2006;49(21): 6177-6196

55. Halgren TA, Murphy RB, Friesner RA, et al. Glide: a new approach for rapid, accurate docking and scoring. 2. Enrichment factors in database screening. J Med Chem. 2004;47(7):1750-1759.

56. Friesner RA, Banks JL, Murphy RB, et al. Glide: a new approach for rapid, accurate docking and scoring. 1. Method and assessment of docking accuracy. J Med Chem. 2004;47(7):1739-1749.

57. Schrödinger. Prime version 3.8, Schrödinger, LLC, New York, NY, 2014.

58. Desmond Molecular Dynamics System, version 4.1, D. E. Shaw Research, New York, NY, 2014. Maestro-Desmond Interoperability Tools, version 4.1, Schrödinger, New York, NY, 2014.

59. Berendsen HJC, Postma JPM, van Gunsteren WF, Hermans J. Interaction models for water in relation to protein hydration. In: Pullman B, editor. Intermolecular Forces. Dordrecht: Reidel; 1981:331-342.

60. Shivakumar D, Williams J, Wu Y, Damm W, Shelley J, Sherman W. Prediction of absolute solvation free energies using molecular dynamics free energy perturbation and the OPLS force field. J Chem Theory Comput. 2010;6(5):1509-1519.

61. Martyna GJ, Klein ML, Tuckerman M. Nosé-Hoover chains: the canonical ensemble via continuous dynamics. J Chem Phys. 1992;97: 2635-2643.

62. Martyna GJ, Tobias DJ, Klein ML. Constant pressure molecular dynamics algorithms. J Chem Phys. 1994;101:4177-4189.

63. Essmann U, Perera L, Berkowitz ML, Darden T, Lee H, Pedersen LG. A smooth particle mesh Ewald method. J Chem Phys. 1995;103(19): 8577-8593.

64. Souers AJ, Leverson JD, Boghaert ER, et al. ABT-199, a potent and selective BCL-2 inhibitor, achieves antitumor activity while sparing platelets. Nat Med. 2013;19(2):202-208.

65. Czabotar PE, Lessene G, Strasser A, Adams JM. Control of apoptosis by the BCL-2 protein family: implications for physiology and therapy. Nat Rev Mol Cell Biol. 2013;15(1):49-63.

66. Wu FE, Gu ZM, Zeng L, et al. Two new cytotoxic monotetrahydrofuran Annonaceous acetogenins, annomuricins A and B, from the leaves of Annona muricata. J Nat Prod. 1995;58(6):830-836.

67. Zeng L, Wu FE, McLaughlin JL. Annohexocin, a novel mono-THF acetogenin with six hydroxyls, from Annona muricata (Annonaceae) Bioorg Med Chem Lett. 1995;5(16):1865-1868.

68. Wu FE, Zeng L, Gu ZM, et al. New bioactive monotetrahydrofuran Annonaceous acetogenins, annomuricin $\mathrm{C}$ and muricatocin $\mathrm{C}$, from the leaves of Annona muricata. J Nat Prod. 1995;58(6):909-915.

69. Zeng L, Wu FE, Oberlies NH, McLaughlin JL, Sastrodihadjo S. Five new monotetrahydrofuran ring acetogenins from the leaves of Annona muricata. J Nat Prod. 1996;59(11):1035-1042.

70. Rieser MJ, Fang XP, Anderson JE, Miesbauer LR, Smith DL, McLaughlin JL. Three new cytotoxic monotetrahydrofuran-ring acetogenins from Annona muricata. Helv Chim Acta. 1993;76(7):2433-2444. 


\section{Publish your work in this journal}

Drug Design, Development and Therapy is an international, peerreviewed open-access journal that spans the spectrum of drug design and development through to clinical applications. Clinical outcomes, patient safety, and programs for the development and effective, safe, and sustained use of medicines are a feature of the journal, which

has also been accepted for indexing on PubMed Central. The manuscript management system is completely online and includes a very quick and fair peer-review system, which is all easy to use. Visit http://www.dovepress.com/testimonials.php to read real quotes from published authors.

Submit your manuscript here: http://www.dovepress.com/drug-design-development-and-therapy-journal 